\title{
Comparison of CDMA and Modulation Schemes for UWB Radio in a Multipath Environment
}

\author{
R. D. Wilson, R. A. Scholtz \\ Communication Sciences Institute \\ University of Southern California \\ Los Angeles CA 90089-2565 \\ robert.wilson@usc.edu,scholtz@usc.edu
}

\begin{abstract}
This paper investigates three different performance metrics for three candidate combinations of multiple access and modulation schemes in ultra wideband radio. The schemes are compared for a single user, multipath channel at a fixed data rate. First, semi-analytic expressions are developed for the probability of error with aribitrary multiple-access coding and amplitude or pulse position modulation, which are then reduced for the special cases of time hopped with bit flipping modulation, time hopped with pulse-position modulation and direct sequence with bit flipping modulation. Using MonteCarlo simulation the bit error rate, probability of outage and probability of best performance are found. The results show that the bit flipped schemes have a consistently lower average bit error rate and outage probabilities and the direct sequence, bit flipped, scheme has highest probability of outperforming both other schemes. In addition, the effect of multipath arrival clustering in channel models was examined and it was found that the same performance curves could be generated with nonclustered arrivals, and hence channel models with non-clustered arrivals can safely be used in performance prediction.
\end{abstract}

\section{INTRODUCTION}

Ultra-wideband radio systems are defined as those where the $10 \mathrm{~dB}$ bandwidth is greater than 0.2 of the center frequency, or where the total bandwidth exceeds $500 \mathrm{MHz}$ [1]. One type of UWB system common in the literature is known as impulse radio, where communication is achieved using short, baseband pulses. Two popularly considered multiple access techniques for an impulse radio system are time hopping (TH), where users are distinguished by their pulse arrival time sequence, and direct sequence (DS) where users are distinguished by their pulse polarity sequence.

A number of researchers have investigated the multipleaccess capacity of an UWB TH system with pulse-position modulation (PPM) [2] [3] [4], and with bit flipping modulation for both direct sequence and time-hopped multiple access [5], using a Gaussian approximation for the inter-symbol interference (ISI) and multiple-access interference. Monte Carlo simulations were used in [6] to calculate the BER of a timehopped M-PAM system under a $\Delta-\delta$ channel model and in [7] to find the average BER of a direct sequence system in

This work was supported in part by the Integrated Media Systems Center, an NSF Research Engineering Research Center, and by the MURI Project under Contract DAAD19-01-1-0477. the presence of multiple access and narrow band interference using measured multipath channels.

In this paper we first develop expressions to calculate the bit error rate of a Rake receiver for time hopped PPM, time hopped bit flipping and direct sequence bit flipping schemes over single user ISI multipath channels. Using these expressions in Monte Carlo simulations not only average bit error rates but also outage probabilities and probability of best performance are compared. Finally, it is shown that the same performance curves can be found by simulation using channel models without clustered arrivals.

\section{UWB SIGNAL MODEL}

The general transmitted UWB waveform of the user, representing the $n^{\text {th }}$ data symbol, is described by

$$
s_{n}\left(d_{n}, t\right)=\sqrt{\frac{E_{s}}{N_{p p s}}} \sum_{k=n N_{p p s}}^{(n+1) N_{p p s}-1} a_{k}^{\left(d s, d_{n}\right)} p\left(t-\tau_{k}^{\left(t h, d_{n}\right)}\right)
$$

where $a_{k}^{d_{n}}$ is the amplitude modulation and $\tau_{k}^{d_{n}}$ the delay on pulse $k$ due to the $n^{\text {th }}$ data symbol $d_{n}$ and the multiple access sequence of the user, $N_{p p s}$ is the number of pulses per data symbol, $p(t)$ is the elemental, unit energy, transmitted UWB pulse function and $E_{s}$ is the energy per symbol. The data and spreading sequence dependent parts of $a_{k}^{\left(d s, d_{n}\right)}$ and $\tau_{k}^{\left(t h, d_{n}\right)}$ can be expressed explicitly as $a_{k}^{d_{n}}=a_{k}^{d s} a_{k}^{d_{n}}$ and $\tau_{k}^{d_{n}}=\tau_{k}^{t h}+\tau_{k}^{d_{n}}=c_{k}^{t h} T_{c}+c_{k}^{d_{n}} \delta_{\text {mod }}$, where $T_{c}$ is called the chip period and $\delta_{\text {mod }}$ is a fixed time delay that can be optimised for the pulse shape $\mathrm{p}(\mathrm{t})$. We will restrict ourselves to binary modulation, i.e., $d_{n} \epsilon\{0,1\}$, and to short code systems, where the spreading sequence period is equal to number of chips per symbol $N_{s s p}=N_{c p s}$.

We will assume a discrete multipath channel without distortion, where the effects of fixed elements such as antennas are implicitly included in the pulse function $p(t)$. The received UWB waveform due to the single transmitted waveform in (1) is

$$
r_{n}\left(u, d_{n}, t\right)=\sum_{i=0}^{L-1} g_{i}(u) s_{n}\left(d_{n}, t-\tau_{i}(u)\right)+w(u, t)
$$




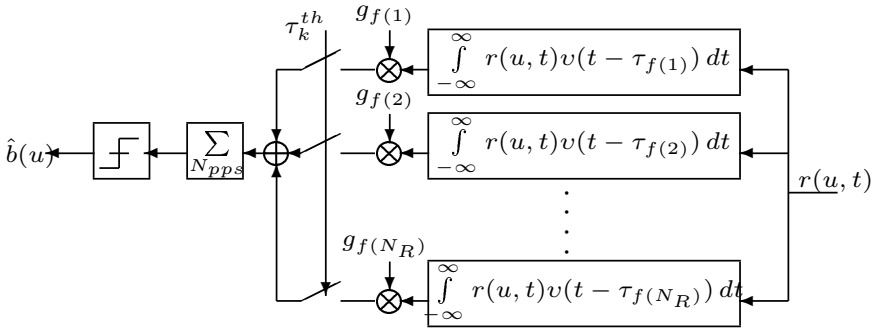

Fig. 1. Receiver model.

where $g_{i}(u)$ is the real amplitude and $\tau_{i}(u)$ the time of arrival of the $i^{t h}$ multipath component and $w(u, t)$ is white gaussian noise with two-sided power spectral density $N_{o} / 2$. Dependence on $u$ indicates a variable is random. We will assume that the channel is stationary over a symbol period. Finally, the observed waveform at time $t$ is the sum of the received waveforms due to all transmissions

$$
r(u, t)=\sum_{n} r_{n}\left(u, d_{n}, t\right) .
$$

\section{Correlation Processing}

The Rake combining technique we will consider here is maximal ratio combining (MRC), where each correlator output is weighted according to its SNR before summing. To simplify the analysis we will assume that the receiver is able to perfectly estimate the channel amplitude parameters and is perfectly synchronised to the received signal, i.e., $g_{i}$ and $\tau_{i}$ are known. The template function for each correlator is given by

$v(\tau)=\frac{1}{\sqrt{N_{p p s}}} \sum_{k=n N_{p p s}}^{(n+1) N_{p p s}-1}\left(a_{k}^{0} \tilde{p}\left(t-\tau-\tau_{k}^{0}\right)-a_{k}^{1} \tilde{p}\left(t-\tau-\tau_{k}^{1}\right)\right)$

and the receiver model is shown in Figure 1.

In general the receiver template version $\tilde{p}(t)$ of the observed pulse $p(t)$ will not be ideal due to distortions occuring in propagation and limitations of implementation. The receiver combines arrivals $f(0)$ to $f\left(N_{R}-1\right)$, which are the $N_{R}$ indices $i$ in (3) with the largest coefficients $g_{i}(u)$. Hence the receiver uses the $N_{R}$ strongest propagation paths for communication.

The output of each Rake finger can be separated into 3 signal and one noise term. The first signal term is due to the pulse to which the receiver is synchronised, the second term is due to the multipath components of the synchronised pulse and the final term is due to multipath components of other pulses.
Under the condition that the time between two consecutive transmitted pulses is equal to at least a pulse length it can be shown that the input to the decision device is $Z(u)=$ $Z_{d}(u)+Z_{m p i}(u)+Z_{i p i}(u)+N(u)$ where

$$
\begin{aligned}
Z_{d}(u)= & \frac{\sqrt{E_{s}}}{N_{p p s}} \sum_{i=0}^{N_{R}-1} \sum_{k=n N_{p p s}}^{(n+1) N_{p p s}-1} g_{f(i)}^{2}(u) a_{k}^{d_{n}} \\
& \times\left(a_{k}^{0} R_{p}\left(\tau_{k}^{0}-\tau_{k}^{d_{n}}\right)-a_{k}^{1} R_{p}\left(\tau_{k}^{1}-\tau_{k}^{d_{n}}\right)\right), \\
Z_{m p i}(u)= & \frac{\sqrt{E_{s}}}{N_{p p s}} \sum_{j=0}^{N_{R}-1} \sum_{\substack{i=0 \\
i \neq j}}^{L-1} \sum_{k=n N_{p p s}}^{(n+1) N_{p p s}-1} g_{f(j)}(u) g_{i}(u) a_{k}^{d_{n}} \\
\times & \left(a_{k}^{0} R_{p}\left(\tau_{f(j)}(u)-\tau_{i}(u)+\tau_{k}^{0}-\tau_{k}^{d_{n}}\right)\right. \\
& \left.\quad-a_{k}^{1} R_{p}\left(\tau_{f(j)}(u)-\tau_{i}(u)+\tau_{k}^{1}-\tau_{k}^{d_{n}}\right)\right),
\end{aligned}
$$

and $Z_{i p i}$ is given in (9) at the bottom of the page. The term $R_{p}(\tau)$ is the cross-correlation between a single received pulse and the template pulse and $N(u)$ is gaussian noise with variance

$$
\sigma^{2}=\frac{N_{o}}{2} \int_{-\infty}^{\infty} h_{n}^{2}(t) d t
$$

Passing the output of the correlator to a thresholding function the probability of receiver error over a given channel is

$$
P_{e}=Q\left(\frac{Z_{d}(u)+Z_{m p i}(u)+Z_{i p i}(u)}{\sigma}\right)
$$

where $Q(x)$ is the Gaussian Integral Function.

\section{A. TH-CDMA}

For time hopping $a_{k}^{d s}=1$ and we let $c_{k}^{t h}$ be the $k^{\text {th }}$ element of the $\left\{N_{c p s}, N_{p p s}, 1\right\}$ difference set.The elements of a difference set are considered a potentially suitable spreading sequence for time hopping UWB systems due to the flatness of the spectrum of such a sequence, which is desirable from the perspective of co-existence with other systems [8]. The possible choices for $v, k$ and $\lambda$ are limited and in later simulations we choose $v=N_{c p s}=993, k=N_{p p s}=32$ and $\lambda=1$.

Pulse position modulation is implemented as $c_{k}^{d_{n}}=(k+$ $\left.d_{n}\right)$ mod 2. Choosing a PPM modulation code as described above, where the number of delayed and non-delayed pulses per symbol is the same for each data bit, ensures that the performance of the receiver is independent of the transmitted data

$$
\begin{array}{r}
Z_{i p i}(u)=\frac{\sqrt{E_{s}}}{N_{p p s}} \sum_{m} \sum_{j=0}^{N_{R}-1} \sum_{\substack{i=0 \\
i \neq j}}^{L-1} \sum_{k^{\prime}=n N_{p p s}}^{(n+1) N_{p p s}-1} \sum_{\substack{k=m N_{p p s} \\
k \neq k^{\prime}}}^{(m+1) N_{p p s}-1} g_{f(j)}(u) g_{i}(u) a_{k}^{d s} a_{k^{\prime}}^{d^{\prime}} a_{k}^{d_{m}}\left(a_{k^{\prime}}^{0} R_{p}\left(\tau_{f(j)}(u)-\tau_{i}(u)+\tau_{k^{\prime}}^{(t h, 0)}-\tau_{k}^{\left(t h, d_{m}\right)}\right)\right. \\
\left.-a_{k^{\prime}}^{1} R_{p}\left(\tau_{f(j)}(u)-\tau_{i}(u)+\tau_{k^{\prime}}^{(t h, 1)}-\tau_{k}^{\left(t h, d_{m}\right)}\right)\right) .
\end{array}
$$


symbol. Such a code is also desirable from a synchronisation perspective [9].

For TH-CDMA and pulse position modulation $Z_{d}$ can be reduced to

$$
\begin{aligned}
Z_{d}(u)=(-1)^{d_{n}} \sqrt{E_{s}} \sum_{j=0}^{N_{R}-1} g_{f(j)}^{2}(u)\left(R_{p}(0)\right. \\
\left.\quad-\frac{R_{p}\left(\delta_{\text {mod }}\right)+R_{p}\left(-\delta_{\text {mod }}\right)}{2}\right) .
\end{aligned}
$$

We will assume that the amplitude of all multipath arrivals are independent and furthermore that they are equally likely to be positive or negative. In this case the $(-1)^{d_{n}}$ factor becomes redundant in $Z_{m p i}(u)$ and we have

$$
\begin{aligned}
Z_{m p i}(u) & =\sqrt{E_{s}} \sum_{j=0}^{N_{R}-1} \sum_{\substack{i=0 \\
i \neq j}}^{L-1} g_{f(j)}(u) g_{i}(u)\left(R_{p}\left(\tau_{f(j)}(u)-\tau_{i}(u)\right)\right. \\
& \left.-\frac{R_{p}\left(\tau_{f(j)}(u)-\tau_{i}(u)+\delta_{m o d}\right)+R_{p}\left(\tau_{f(j)}(u)-\tau_{i}(u)-\delta_{m o d}\right)}{2}\right) .
\end{aligned}
$$

Due to the fundamental property of $\{v, k, 1\}$ difference sets, in the expression for $Z_{i p i}$ of (9) at most one term in the double sum over $k$ and $k^{\prime}$ is non-zero. With some simplification we have

$$
\begin{aligned}
Z_{i p i}(u)=\frac{\sqrt{E_{s}}}{N_{p p s}} \sum_{j=0}^{N_{R}-1} \sum_{\substack{i=0 \\
\alpha_{j, i} \neq 0}}^{L-1} g_{f(j)}(u) g_{i}(u)\left(R_{p}\left(\beta_{j, i}(u)\right)\right. \\
\left.-\frac{R_{p}\left(\beta_{j, i}(u)+\delta_{\text {mod }}\right)+R_{p}\left(\beta_{j, i}(u)-\delta_{\text {mod }}\right)}{2}\right)
\end{aligned}
$$

where $\alpha_{j, i}=\left[\frac{\tau_{f(j)}(u)-\tau_{i}(u)}{T_{c}}\right], \beta_{j, i}(u)=\tau_{f(j)}(u)-\tau_{i}(u)-$ $\alpha_{j, i} T_{c}$ and $[x]$ meaning the nearest integer to $x$.

For bit flipping modulation $a_{k}^{d_{n}}=(-1)^{d_{n}}$ and the terms of the correlator output are

$$
\begin{aligned}
Z_{d}(u) & =(-1)^{d_{n}} \sqrt{E_{s}} \sum_{j=0}^{N_{R}-1} g_{f(j)}(u)^{2} 2 R_{p}(0) \\
Z_{m p i}(u) & =\sqrt{E_{s}} \sum_{j=0}^{N_{R}-1} \sum_{\substack{i=0 \\
i \neq j}}^{L-1} g_{f(j)}(u) g_{i}(u) 2 R_{p}\left(\tau_{f(j)}(u)-\tau_{i}(u)\right)
\end{aligned}
$$$$
Z_{i p i}(u)=\frac{\sqrt{E_{s}}}{N_{p p s}} \sum_{j=0}^{N_{R}-1} \sum_{\substack{i=0 \\ \alpha_{j, i} \neq 0}}^{L-1} g_{f(j)}(u) g_{i}(u) 2 R_{p}\left(\beta_{j, i}(u)\right)
$$

In comparing the $Z_{d}$ components of PPM and bit flipping modulation in (15) and (12) respectively, note that $R_{p}(0)>$ $-\frac{R_{p}\left(\tau_{m o d}\right)+R_{p}\left(-\tau_{\text {mod }}\right)}{2}$, so bit flipping modulation has an inherent advantage in signal separation over PPM modulation and will give better performance in the absence of multipath. However the difference between the multipath and inter-pulse interference terms is not so obvious and will depend on the channel and signal shape.

\section{B. DS-CDMA}

For direct sequence we will consider bit flipping modulation only. The $Z_{d}(u)$ and $Z_{m p i}(u)$ terms of the correlator output are independent of the multiple access scheme and therefore are the same as (15) and (16) for the time hopped bit flipping case. For the $Z_{i p i}(u)$ term because there is a pulse present in every chip interval every channel path causes interference to the reception of every pulse. Once again we can assume $d_{m}=0$ without loss of generality and $Z_{i p i}(u)$ becomes

$$
\begin{aligned}
Z_{i p i}(u)=\frac{\sqrt{E_{s}}}{N_{p p s}} \sum_{j=0}^{N_{R}-1} \sum_{\substack{i=0 \\
\alpha_{j, i} \neq 0}}^{L-1} g_{f(j)}(u) g_{i}(u) 2 R_{p}\left(\beta_{j, i}\right) \\
\times \sum_{k^{\prime}=n N_{p p s}}^{(n+1) N_{p p s}-1} a_{k^{\prime}}^{d s} a_{k^{\prime}+\alpha_{j, i}}^{d s}
\end{aligned}
$$

where $k^{\prime}+\alpha_{j, i}$ is calculated mod $N_{p p s}$ and $\alpha_{j, i}$ is defined as before. Note that for sequences with good auto-correlation properties the summation over $k^{\prime}$ is small, in particular in this case we will use length 1023 Gold codes for which the sum is -1 , so (18) takes the same form as (17) for time hopped bit flipping. Note however that in the case of direct sequence $N_{p p s}=N_{c p s}$ which is typically much larger than $N_{p p s}$ in the time hopping system, therefore we expect lower variance in $Z_{i p i}$ for direct sequence coding than for time hopped.

\section{Simulation Method}

The performance of each of the above schemes was simulated by generating sample propagation channels and calculating the probability of error according to (11). The channel model is that recommended by the IEEE 802.15.3a channel modelling sub-committee for use in evaluation of UWB PHY submissions [10]. The impulse response can be written as

$$
c(t)=X(u) \sum_{n=0}^{N-1} \sum_{k=0}^{K-1} g_{n, k}(u) \delta\left(t-\tau_{n, k}(u)\right)
$$

where $\tau_{n, k}=T_{n}+\zeta_{n, k}$ is the time of arrival and $g_{n, k}=$ $p_{n, k} \xi_{n} \beta_{n, k}$ is the amplitude coefficient of the $k^{t h}$ ray in the $n^{t h}$ cluster. The clusters and intra-cluster rays have exponentially distriubted inter-arrival times and the fading is lognormal. The polarity of each arrival is determined by $p_{n, k}$ and is \pm 1 with equal probability. For any given channel realisation the total energy in the double sum over $g_{n, k}$ is normalised to unity and the entire impulse response multiplied by the lognormal shadowing term $\mathrm{X}(\mathrm{u})$.

In [10] the authors suggest four sets of parameters that have been found to capture some of the key characteristics of observed UWB multipath channels. The models denoted CM1, 
TABLE I

Channel Characteristics

\begin{tabular}{|l|c|c|c|}
\hline \hline Channel characteristic & CM 1 & CM 3 & CM 4 \\
\hline Mean delay spread (ns) & 5 & 15.9 & 29.9 \\
\hline RMS delay spread (ns) & 5 & 15 & 26 \\
\hline NP (85\%) & 22 & 84 & 193.7 \\
\hline
\end{tabular}

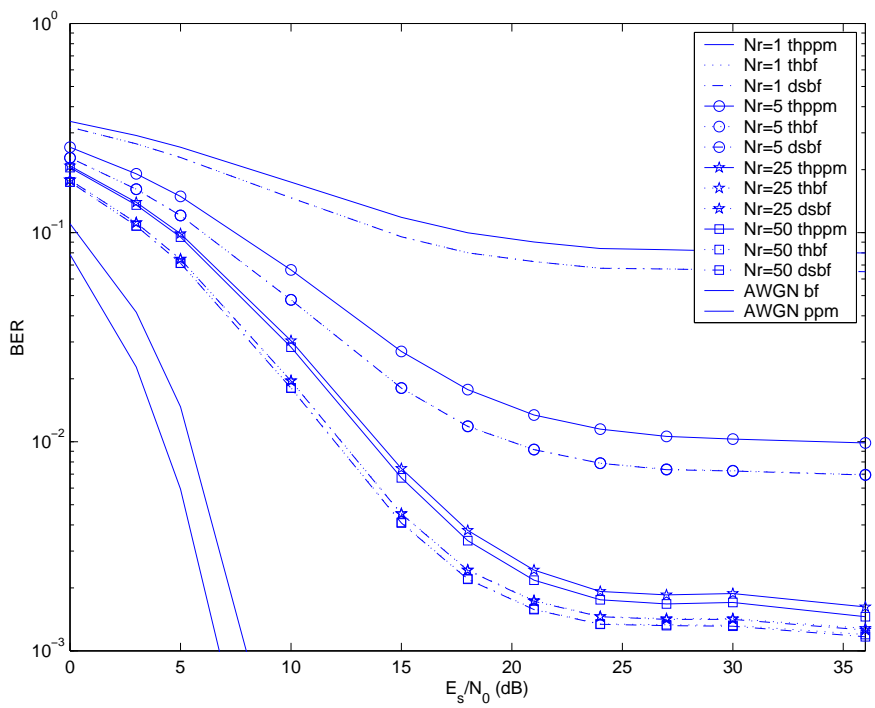

Fig. 2. BER performance of different schemes under multipath channel model CM1.

CM3 and CM4 are used here and their channel characteristics are given in Table I. The channel characteristic NP $(85 \%)$ is the number of paths capturing $85 \%$ of the energy in the channel and the mean and rms delay spreads are defined in the usual way.

The chip period was fixed at $T_{c}=5 \mathrm{~ns}$, the pulse duration is $T_{p}=1 \mathrm{~ns}$ and $p(t)=\sqrt{2} \sin \left(2 \pi t / T_{p}\right)$ with support $0 \leq t<T_{p}$. For the monocycle pulse function used here the optimal delay for pulse position modulation is $\delta_{\text {mod }}=$ $T_{p} / 2$, when $-\frac{R_{p}\left(\delta_{\text {mod }}\right)+R_{p}\left(-\delta_{\text {mod }}\right)}{2}=\frac{1}{2} R(0)$. As discussed in section III-A, this is less than the $R(0)$ which appears in the equivalent expression for bit flipping modulation and results in an inherent $1.25 \mathrm{~dB}$ advantage when using bit flipping.

\section{RESUlts}

Figure 2 shows the average bit error rates for communication over channels of type CM1 against signal to noise ratio for different numbers of Rake fingers in the receiver, with the correlation receiver performance in a single path AWGN channel shown for reference.

Bit-flipping acheives lower average bit error rates over the entire range of the curve, and at low SNR in particular they show the expected $1.25 \mathrm{~dB}$ advantage over pulse position modulation. At the low SNR end of the curves we see about $5 \mathrm{~dB}$ gain in going from 1 to 5 correlators in the receiver, a $2.5 \mathrm{~dB}$ gain in going from 5 to 25 correlators and $0.2 \mathrm{~dB}$ gain between 25 and 50 correlators. Over the same range at the high SNR end the error floor is lowered almost two

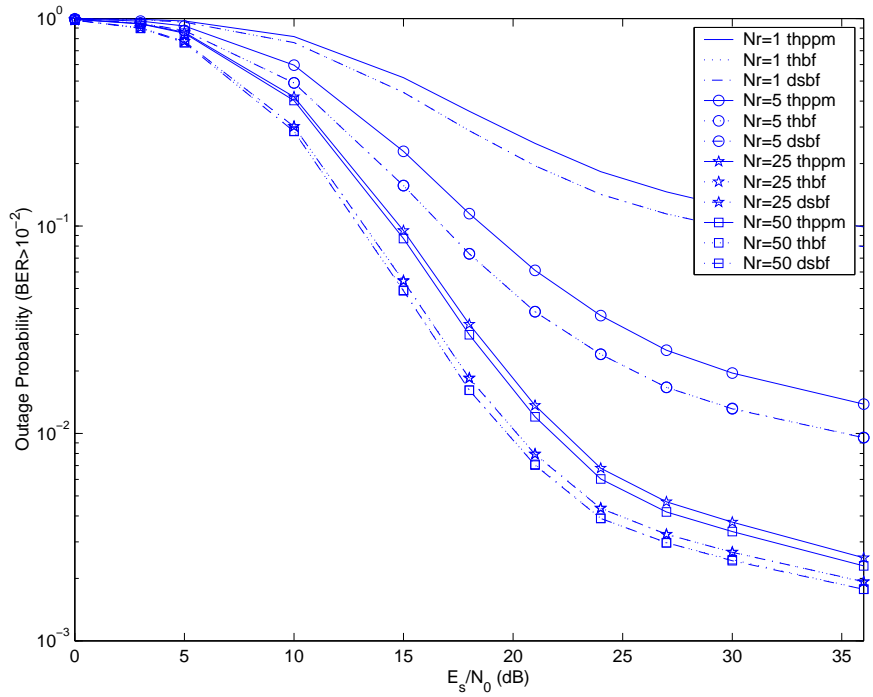

Fig. 3. Outage probabilities of different schemes under multipath channel model CM1.

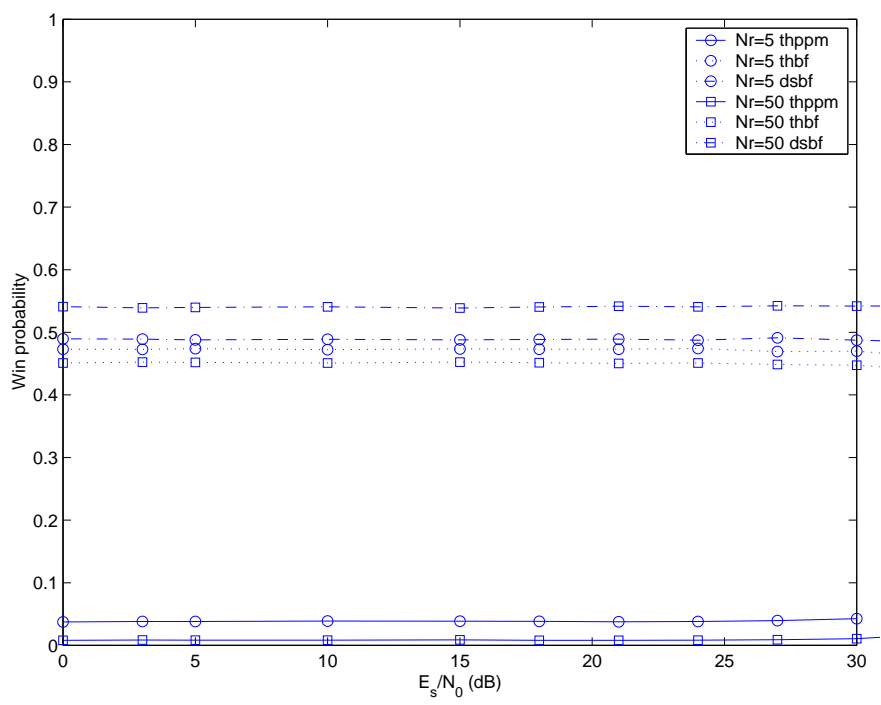

Fig. 4. Probability of acheiving the best BER over a given channel for each scheme over CM1.

orders of magnitude from near 0.08 to below 0.002 . Figures 3 and 4 show the outage probability and probability of win respectively for channel model CM1. Note that although the time-hopped and direct-sequence bit-flipping regimes are indistinguishable in terms of average bit error rate and outage probability, the direct sequence technique has a higher win probability due to its lower variance in $Z_{i p i}(u)$. The gap in win probability between the schemes also increases when more Rake correlators are used.

Figure 5 compares the average bit error rate over channel model CM1 to that over channel model CM4 using a 5 correlator Rake. In CM4 the channel energy is being spread over more paths, corresponding to lower mean energy per path and an increase in BER at low signal to noise ratio, while at high SNR the interference power is lower, delaying the onset 


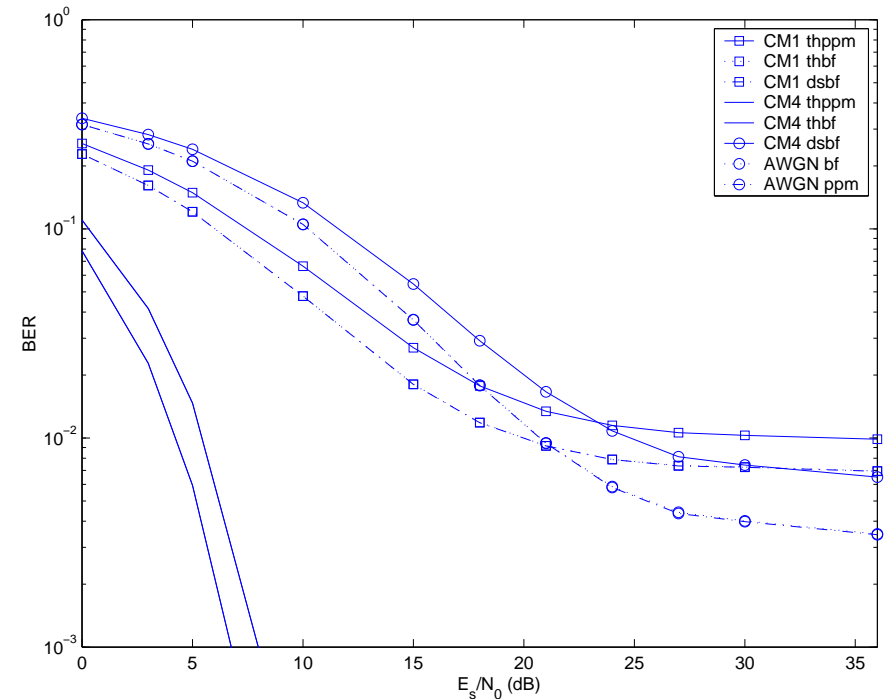

Fig. 5. Comparison of BER performance using models CM1 and CM4.

TABLE II

NON-CLUSTERED CHANNEL CHARACTERISTICS

\begin{tabular}{|l|c|}
\hline \hline Channel characteristic & NC \\
\hline Mean delay spread (ns) & 10 \\
\hline RMS delay spread (ns) & 10 \\
\hline NP $(85 \%)$ & 87.5 \\
\hline
\end{tabular}

of the error floor.

In Figure 6 the average bit error rate over channel CM3 is shown with the same measure over a channel without clustering. That is, the inter-arrival times were chosen according to a simple exponential distribution and the parameters of the arrival time and log-normal fading distributions were optimised to best fit the performance curves for the CM3 channel model. The channel characteristics are shown in Table II. The bit error rate curves over the different channels are nearly identical and the other performance curves show a similarly close fit.

\section{CONClusions}

The average bit error rate and outage probability performance of three UWB multiple access and modulation combinations have been found for a single user, using the channel models for recommended for use in IEEE 802.15.3a evaluations.

As expected, bit flipped modulation demonstrated better performance than pulse position modulation in terms of average BER and outage probability due to it's lower correlation between symbols. It was expected that direct sequence coding should have an advantage over time hopping due to lower variance interpulse interference, but this advantage was not noticeable in terms of average BER or outage probability. However, direct sequence multiple access coding was more likely to acheive the lowest BER for a fixed channel.

Finally, a comparison was made between using channel models with clustered arrivals versus one with no clustering. It

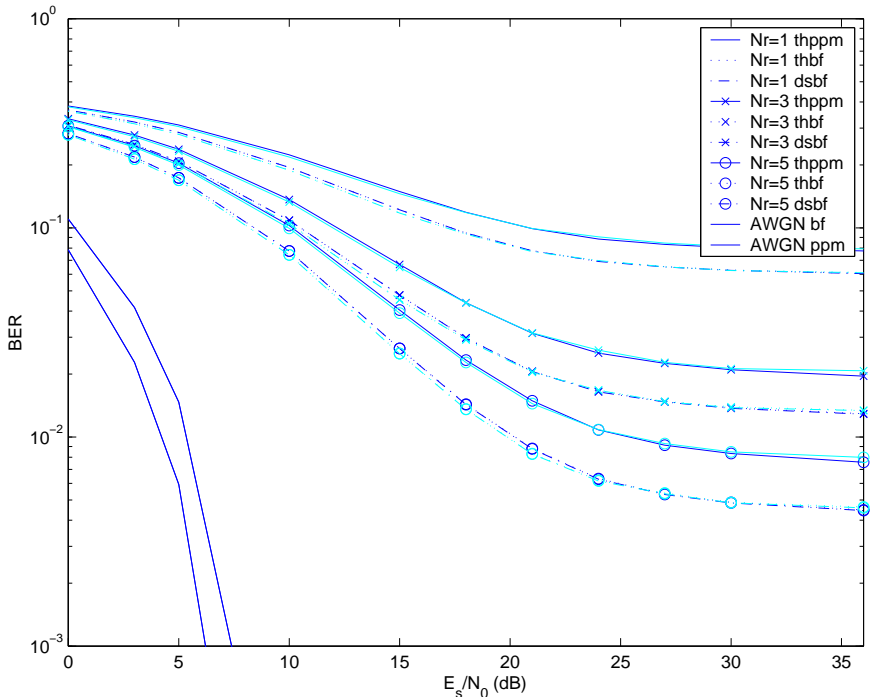

Fig. 6. Comparison of BER performance using model CM3 (dark line) and the non-clustered model (light line).

was found that with proper selection of parameters the models can be made equivalent in an average BER, outage probability and "win" probability sense. The ability to use equivalent nonclustered channels to analyse radio performance should make theoretical problems more tractable.

\section{REFERENCES}

[1] Federal Communications Commission, "Revision of part 15 of the commission's rules regarding ultra-wideband transmission systems: First report and order," April 2002. ET-Docket 98-153.

[2] R. A. Scholtz, "Multiple access with time-hopping impulse modulation," in Proc. MILCOM, vol. 2, pp. 447-450, 1993.

[3] M. Z. Win and R. A. Scholtz, "Ultra-wide bandwidth time-hopping spread-spectrum impulse radio for wireless multiple access communications," Trans. on Communications, vol. 48, pp. 679-689, Apr. 2000.

[4] A. Taha and K. M. Chugg, "Multipath diversity reception of wireless multiple access time-hopping digital impulse radio," in Proc. UltraWideband Systems and Technology, pp. 283-287, IEEE, 2002.

[5] V. S. Somayazulu, "Multiple access performance in UWB systems using time hopping vs. direct sequence spreading," in Proc. WCNC, pp. 522525, IEEE, 2002.

[6] J. R. Foerster, "The effects of multipath interference on the performance of UWB systems in an indoor wireless channel," in Proc. Vehicular Technology Conference, pp. 1176-1180, IEEE, 2001.

[7] J. R. Foerster, "The performance of a direct-sequence spread ultrawideband system in the presence of multipath, narrowband interference, and multiuser interference," in Proc. Ultra-Wideband Systems and Technology, pp. 87-92, IEEE, 2002.

[8] R. A. Scholtz, P. V. Kumar, and C. J. Corrado-Bravo, "Signal design for ultra-wideband radio," in SETA, 2001.

[9] B. Sklar, Digital Communications: Fundamentals and Applications, ch. 10.2.2. Prentice Hall, 2001.

[10] J. R. Foerster, "Channel modeling sub-committee report (final)," Tech. Rep. P802.15-02/368r5-SG3a, IEEE P802.15 Working Group for Wireless Personal Area Networks (WPANs), December 2002. http://grouper.ieee.org/groups/802/15/pub/2002/Nov02/02490r0P80215_SG3a-Channel-Modeling-Subcommittee-Report-Final.zip. 